\title{
CONFÉRENCE
}

\section{Atrophie maxillaire : Ostéotomie de LEFORT I et greffe osseuse associée en chirurgie pré-implantaire.}

\section{Pr Raoul G, Dr Lauwers L, Pr Ferri J} CHRU Lille

Les patients présentant des atrophies maxillaires majeures restent un challenge en réhabilitation fixe. La fonte maxillaire entraine un décalage des bases osseuse avec un recul maxillaire aggravé par la perte de dimension verticale, et l'autorotation mandibulaire, elle aussi souvent atrophique.

Les prothèses adjointes deviennent alors très volumineuses et instables. La solution implanto-portée est alors étudiée pour assurer la stabilisation et la rétention. II faut résoudre un double problème : hauteur faciale et décalage antéropostérieur.

L'ostéotomie de Lefort I d'avancée et descente répond à cette demande, transférant la région prémolaire en canine et permettant de greffer très généreusement le bas-fond sinusien.

Cette étape est d'ailleurs compatible avec le traitement des éventuelles sinusites et communications buccosinusiennes secondaires au passif alvéolodentaire du patient.

Des greffes vestibulaires complémentaires sont éventuellement associées. Le choix du site donneur sera alors sujet à discussion.

Face à ce long et lourd protocole s'étendant au moins une année, une alternative par implants zygomatiques est entre-autres envisageable mais avec un concept prothétique différent.

Nous n'aborderons pas les techniques d'ostéotomie et réhabilitation mandibulaire qui doivent néanmoins faire partie du plan de traitement global. L'ensemble de ces éléments fait partie de la stratégie de réhabilitation habituellement discutée en concertation pluridisciplinaire.

gwenael.raoul@gmail.com

(C) The authors, published by EDP Sciences. This is an Open Access article distributed under the terms of the Creative Commons Attribution License 4.0 (http://creativecommons.org/licenses/by/4.0/). 